\title{
2D- STI Quantitative Analysis of PCI Postoperative Patients with the Myocardial Perfusion Change and its Clinical Significance
}

\author{
Shujing Wei ${ }^{1, a,{ }^{*}}$ \\ ${ }^{1}$ Department of Ultrasound, the First People's Hospital of Nanning, Nanning, 530022, China. \\ ashuingwei@126.com \\ ${ }^{*}$ Corresponding author shuingwei@126.com
}

\begin{abstract}
Keywords: 2D- STI technology; Coronary heart disease; PCI surgery; Myocardial perfusion
\end{abstract}
\begin{abstract}
Objective: To observe and study 2D- STI technique to investigate the coronary artery disease after PCI in patients with preoperative left ventricular myocardial systolic function improvement, and it is provided the clinical to evaluate the effect of PCI surgery more accurate quantitative indicators. Methods: The heart patients of our hospital with a total of 50 patients with myocardial infarction since 2011, aged 40 to 70 years old, and successfully implement PCI treatment, were randomly divided into two groups: PCI preoperative room wall segment is defined as the preoperative group, postoperative ventricular wall segment after 5 days is defined as the postoperative group. 50 cases of our hospital outpatient patients are as the control group. Both groups in the PCI preoperative $1 \sim 3$ days, PCI postoperative five days and the control line echocardiography respectively. Results: In the PCI preoperative 1-3 days and PCI postoperative application of 5 days 2D-STI of abnormal segmental left ventricular wall of PSS, PSSR, RS and CS, and combined with myocardial acoustic imaging before PCI, the results of the electrocardiogram and myocardial enzymologist to the quantitative analysis of myocardial perfusion, the results showed that the PSS, PSSR of perfusion improved segmental radial and tangential strain were significantly improved, the infusion does not improve segment, there are no changes in the index. Conclusion: 2D- STI technology is from the Angle of restriction, can real-time tracking trajectories of myocardial at any location, and more accurate measurements, the application of 2D-STI technology is to provide the clinical to evaluate the effect of PCI surgery more accurate quantitative indicators, and it is with broad clinical application value.
\end{abstract}

\section{Introduction}

Two-dimensional speckle tracking imaging (2D- STI) is for detecting myocardial strain and strain rate, etc, on the mechanical properties of the heart in the cardiac cycle qualitative and quantitative description, no Angle dependence and less affected by the surrounding tissue and motion. Ultrasonic two-dimensional speckle tracking technology is the emergence of a new technology in recent years, can obtain three direction of each segment myocardial strain value: longitudinal strain values (LS), radial strain values (RS), circumferential strain values (CS), to overcome before the application of ultrasonic tissue imaging technique can only evaluate the longitudinal myocardial strain and obvious Angle dependent has its limitations. Strain (S) is the quantitative evaluation of myocardial movement index, reflects the degree of myocardial fibbers after stress deformation, can use the formula $\mathrm{S}=(\mathrm{L}-\mathrm{L} 0$ )/L0 says (L0 says initial length, L said after deformation length). At present, the research shows that the technology can quantitatively evaluate the degree of ventricular wall motion abnormalities in patients with, but for different parts of myocardial infarction, cardiac muscle each section three less strain value change rule of the comparison of the similarities and differences. For the patients in our hospital since 2011 heart patients with a total of 50 patients with myocardial infarction, observation and evaluation of 2DSTI technique to investigate the coronary artery disease after PCI in patients with preoperative left ventricular myocardial systolic function improvement, now report as follows. 


\section{Materials and Methods}

\section{Materials}

For the patients in our hospital since 2011 heart patients is with a total of 50 patients with myocardial infarction (mi), 28 cases of male, female 22 cases, aged 40 to 70 years old, average age (57.6 \pm 8.5$)$, and successfully implement PCI treatment, were randomly divided into two groups: PCI preoperative room wall segment is defined as preoperative group, postoperative ventricular wall segment is defined as a group after 5 days. 50 cases of our hospital outpatient patients as control group, 29 patients are the male, and 21 patients are the female, aged 40 to 70 years old, average age (58.2 \pm 7.6 ), on checking out heart disease, and $2 \mathrm{~d}$ image satisfaction. All the subjects are in sinus rhythm, no complete left bundle branch block.

\section{Methods}

The application of the Dutch Philips IE33 color ultrasonic diagnostic instrument production company, M3S ultrasound probe, the frequency of 1.0-3.0 MHz. Client to take the left side, PCI preoperative, PCI postoperative $1 \sim 3$ days for five days and the control group respectively echocardiography, synchronous record of chest lead electrocardiogram, after waiting for images show satisfaction, instruct patients breathe out at the end of the breathless, sampling left ventricular short axis valve, papillary muscle and apex level two-dimensional gray-scale dynamic figure $(50 \sim$ 70 frames/s) frame frequency, stable take three consecutive images of the cardiac cycle. Image after weaning, USES the QLAB8.0 software into TMQA plug-in, the system automatically will be 18 segmental left ventricular short axis points, for each section curve of corresponding parameters, analysis of the abnormal segmental left ventricular wall PSS, PSSR, RS and CS, to evaluate the effect of reperfusion therapy.

\section{The oservation indicators}

In the PCI preoperative 1-3 days and PCI postoperative application of 2D- STI five days of abnormal segmental left ventricular wall of PSS, PSSR, RS and CS, and combined with myocardial acoustic imaging before PCI, the results of the electrocardiogram (ecg) and myocardial enzymology to quantitative analysis of myocardial perfusion..

\section{Statistical processing}

With mathematical statistics software SPSS19.0 for data collection and statistical analysis, and chi-square test, $\mathrm{P}<0.05$, significant difference has statistical significance.

\section{Results}

The results showed that the PSS of perfusion improved segmental, PSSR radial and tangential strain were significantly improved, infusion did not improve the section, there were no changes in the index. At the same time predict left ventricular global systolic function improved and left ventricular remodelling. 2D- STI technology is from the Angle of restriction, can real-time tracking trajectories of myocardial at any location, and more accurate measurements, STI technology application, to provide clinical to evaluate the effect of PCI surgery more accurate quantitative indicators. This inspection method is relatively simple, non-invasive and repeatable track observation, and sensitive to found the presence of myocardial ischemia and myocardial infarction, obviously increase the diagnostic rate of coronary heart disease has wide clinical application value. See Table 1, Fig.1 and Fig.2. 
Table 1: 2D- STI measured parameters between the case group and normal control group

\begin{tabular}{|c|c|c|c|c|c|}
\hline \multirow[b]{2}{*}{$\begin{array}{c}\text { Variabl } \\
\text { es }\end{array}$} & \multirow{2}{*}{$\begin{array}{c}\text { Norm } \\
\text { alcontrols } \\
(\mathrm{n}=50)\end{array}$} & \multicolumn{4}{|c|}{ Patients $(n=50)$} \\
\hline & & Preoperaive & $\mathrm{P}$ & $\begin{array}{c}\text { five days } \\
\text { after }\end{array}$ & $\mathrm{P}$ \\
\hline PSS & $26.92 \pm 3.58$ & $12.36 \pm 1.72$ & $\begin{array}{c}0.03 \\
2\end{array}$ & $11.28 \pm 1.78$ & $\begin{array}{c}0.02 \\
7\end{array}$ \\
\hline PSSR & $1.62 \pm 0.12$ & $0.82 \pm 0.12$ & $\begin{array}{c}0.02 \\
4\end{array}$ & $0.65 \pm 0.38$ & $\begin{array}{c}0.00 \\
6\end{array}$ \\
\hline $\mathrm{RS}$ & $-2.12 \pm 0.08$ & $-0.91 \pm 0.11$ & $\begin{array}{c}0.01 \\
6\end{array}$ & $-0.76 \pm 0.14$ & $\begin{array}{c}0.01 \\
4\end{array}$ \\
\hline $\mathrm{CS}$ & $-2.05 \pm 0.18$ & $-0.86 \pm 0.19$ & $\begin{array}{c}0.01 \\
5\end{array}$ & $-0.82 \pm 0.17$ & $\begin{array}{c}0.01 \\
6\end{array}$ \\
\hline
\end{tabular}

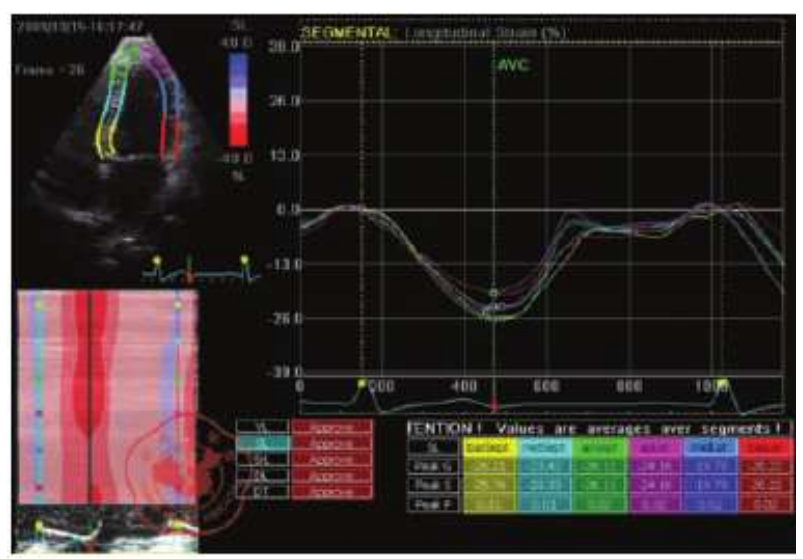

Fig. 1 The normal left ventricular apex four cavity longitudinal strain curve

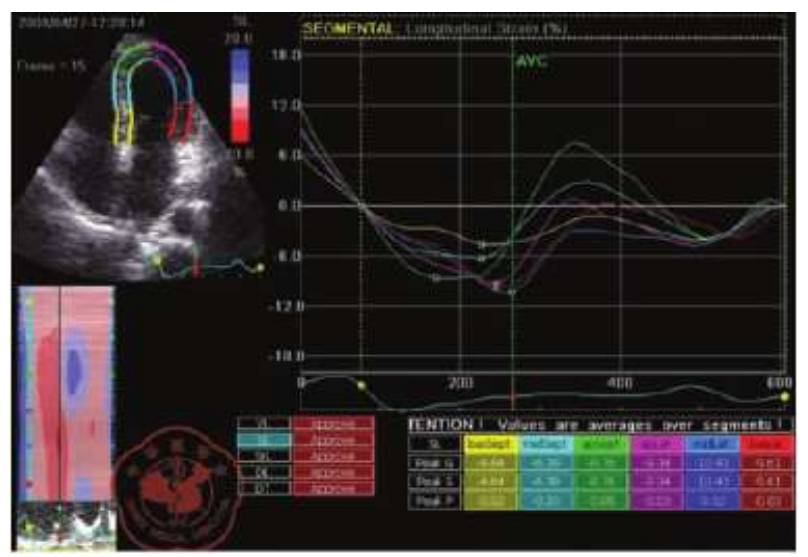

Fig. 2 PCI in patients with myocardial infarction preoperative left ventricular apex four cavity longitudinal strain curve

\section{Discussion}

\section{The incidence}

Non ST-elevation acute myocardial infarction and unstable angina patients with coronary artery lesions tend to be heavy, often appear more vascular or left main lesion, clinical often combine multiple risk factors. Myocardial infarction is a common clinical critical patient, patients with serious threats to life and influence the quality of life, early and accurate diagnosis of myocardial infarction, range, degree and the changes of cardiac function is important in establishing treatment strategies and prognosis. At present, the evaluation of ventricular wall motion abnormalities in patients with myocardial infarction, cardiac function method, the visual two-dimensional ultrasound 
images is still the most widely clinical application method, but its are greatly influenced by subjective factors. Because of endocrinal myocardial muscle bundle spiral and middle anatomical characteristics of circular beam, myocardial movement including the longitudinal, is the radial and circumferential movement. Ultrasound speckle tracking technology based on two-dimensional ultrasound image, on the basis of real-time tracking frequency between different frames in the same position of myocardial space trajectory, overcome the ultra sound beam and the limitation of myocardial motion Angle, it can measure the longitudinal, radial and circumferential strain values of myocardial motion quantitatively evaluation local myocardial motion state and overall cardiac function in many aspects.

Studies have reported that myocardial infarction segment longitudinal, radial and circumferential strain values than normal control group were significantly reduced. This research shows that, compared with normal group, two longitudinal strain values not only in myocardial infarction group section to reduce myocardial infarction, and adjacent segmental apart is also reduced. Abroad such as Raymond animal experiment results are similar to this research conclusion. Produce this kind of phenomenon is not clear, that may be related to the longitudinal strain value is mainly influenced by the endocrinal myocardial fibers, and the middle of the back lining of myocardial ischemia is the earliest involvement on more easily also. In addition, after myocardial infarction, the infarction area is the main part of the left ventricular remodeling. Kim's research report, in the process of myocardial remodeling, when load capacity, room wall tension increased, ventricular geometry change can cause the longitudinal strain values. As a result, the segment longitudinal strain value to reduce myocardial infarction may also rather than to increase the quantity of cardiac, vicarious expansion. But this study showed that myocardial infarction segments than in myocardial infarction segments more significant the longitudinal strain values, tip longitudinal strain value can not only quantitatively evaluate regional ventricular wall motion wanes, a good indicator of the section is also accurate positioning myocardial infarction.

Two-dimensional speckle tracking imaging techniques (2D-STI) is a newly developed a kind of objective and quantitative, real-time and non-invasive new method to evaluate the local myocardial function, can evaluate the papillary muscle deformation from multiple directions. In recent years, the incidence of AMI is on the rise, timely, fully and continuously opening blood vessels in the related parts of the infarction is to save the dying myocardium, reduce infarction size, improve prognosis, the key to reduce the case fatality rate. AMI due to myocardial ischemia injury after the whole left ventricular infarction area and the infarction area shape and the size of the change is called the left ventricular remodeling, its foundation for cell including loss of cardiac muscle cells, myocardial cells can't adjust the benign hypertrophy and fibrosis of extra cellular matrix. Due to the redistribution of cardiac ischemia and heart working load, lead to the myocardial infarction area load increase, causes the left ventricular remodeling, can be divided into early infarction of exhibition (infarct expansion, IE) and ventricular late overall expansion (global ventricular dilatation, GVD (gnu visual debugger)). IE is in the absence of further myocardial necrosis, infarction area disproportionately thin and elongated, room wall deformation and local lumen enlargement, rather than the infracted myocardium was not involved in the process, mainly in infarction after hours to 6 weeks. GVD (gnu visual debugger) refers to the left ventricular contraction, increased end-diastolic volume and left ventricular elliptical form loss and change the ball samples, progressive HBI called late sustainable from 6 weeks to 1 year. As the important index of determining is the cardiac function and long-term prognosis of patients with MI. White research thinks, LVEDV, LVESV and LVEF can be sensitive to the variation of the left ventricular structure and function after AMI, including LVESV is the most effective predictor.

Ultrasonic testing of left ventricular function changes and ventricular wall movement disorders can accurately reflect the state of myocardial blood supply, is a good indicator of blood vessel revascularization prognosis evaluation. Has confirmed that early after AMI $(<12 \mathrm{~h})$ revascularization IRA returned to effective forward flow, can reduce AMI area, thereby reducing ventricular remodeling, protecting heart function and improve the long-term prognosis of AMI. At present, the clinical using traditional $2 \mathrm{~d}$ and $\mathrm{M}$ type echocardiography evaluation of reperfusion 
after curative effect still is given priority to with visual, influence factors is more, the missed diagnosis is high, compared to the DTI in this aspect is more sensitive and specific. Have a large number of clinical studies confirmed that the tissue velocity, strain and strain rate imaging technology can be more sensitive and accurate evaluation of ventricular systolic and diastolic function, especially suitable for evaluation of segmental myocardial function. But its Angle dependency problem that allow not to ignore, recently, according to the spot tracking technology of speckle tracking imaging greatly make up for the defects, which can get more information about the myocardial deformation in great quantities, enable us to more comprehensive, rapid, sensitive and accurate for evaluation of cardiac function. Also called two-dimensional speckle tracking imaging strain echocardiography (two - dimensional strain echocardiography, 2 dse arithmetic) is mainly through to the high frame frequency two-dimensional image spot echoes with track frame by frame, each track spots was the echo of about $20 \sim 40$ pixels, throughout the cardiac cycle together they can follow the myocardial tissue around the movement to change in position, so the distance between the adjacent two spots echo relative change, on the other hand, reflects the local myocardial systolic and diastolic (that is, two-dimensional strain and strain rate), which can be calculated and graphical myocardial velocity and deformation. Existing research is by using MRI or sonar micro measurement technology confirmed using two-dimensional strain echocardiography for measuring by normal, ischemic myocardial strain and strain rate on the accuracy of the results, and by the strain, strain rate results with TDI. This study first conducted a STI and consistency analysis of tissue measurement results, shows both show good consistency, once again confirm the accuracy of the measurement result. And then by PCI reperfusion therapy for early stage of AMI patients in acute period application STI peak shrinkage strain and strain rate analysis, found that compared with control group, the group of patients with myocardial infarction despite improved myocardial perfusion, overall systolic function (LVEF) is normal, but still left ventricular myocardial deformation of each section of different level decreased, compared with peak contraction is more sensitive to strain rate can reflect the function of the myocardial damage. Commonly used indicators reflect the left ventricular remodeling in echocardiography including LVESV, LVEDV, WMSI and LVEF, myocardial infarction patients in this study, although the early intervention treatment actively, but such indicators are still significant differences with the control group, the prompt still occurs early left ventricular remodeling is the IE. Further correlation analysis also suggests that peak systolic total strain and strain rate associated with LVESV, WMSI and LVEF is good, so it can reflect the whole left ventricular systolic function, and the degree of left ventricular remodeling.

\section{Conclusion}

The 2D- STI quantitative analysis of myocardial perfusion in patients with PCI postoperative is to evaluate the effect of myocardial reperfusion therapy, at the same time to predict the improvement of the whole left ventricular systolic function and left ventricular remodeling. Through the comprehensive index, Cox statistical model is set up, forming a reliable, non-invasive and repeatable, convenient and accurate method to evaluate the therapeutic effect of PCI postoperative provides more accurate quantitative indicators, improve level and innovation ability of diagnosis and treatment of cardiovascular disease, and the expectations for the domestic leading level.

\section{Acknowledgement}

This research was financially supported by the National Science Foundation.

\section{References}

[1] Eeckhout E, Kern MJ. The coronary no-reflow phenomenon: a review of mechanism and therapies. European hesrt journal. 2011. 
[2] Fuster V, Badimon L, Badimon JJ,et al. The pathogenesis of coronary artery disease and the acute coronary syndromes:part 1. New England Journal of Homeopathy. 2012.

[3] Ito H, Maruyama A,Iwakura K,et al. Clinical implications of the no-reflow phenomenon: a predictor of complications and left ventricular remodeling in reperfused anterior wall myocardial infarction. Circulation. 2013.

[4] Sakuma T,Leong-Poi H, Fisher N G, et al. Further insights into the no-reflow phenomenon after primary angioplasty in acute myocardial infarction: the role of microthromboemboli. Journal of the American Society of Echocardiography. 2012.

[5] Eeckhout E, Kern MJ. The coronary no-reflow phenomenon: a review of mechanisms and therapies. European Heart Journal. 2012.

[6] Poli A, Fetiveau R, Vandoni P, et al. Integrated analysis of myocardial blush and ST-segment elevation recovery after successful primary angioplasty: Real-time grading of microvascular reperfusion and prediction of early and late recovery of left ventricular function. Circulation. 2012.

[7] Stone GW, Peterson MA, Lansky AJ, et al. Impact of normalized myocardial perfusion after successful angioplasty in acute myocardial infarction. Journal of the American College of Cardiology. 2013.

[8] Abbo KM, Dooris M, Glazier S, et al. Features and outcome of no-reflow after percutaneous coronary intervention. The American Journal of Cardiology. 2012.

[9] Lee DP, Herity NA, Hiatt BL, et al. Adjunctive platelet glycoprotein IIb/IIIa receptor inhibition with tirofiban before primary angioplasty improves angiographic outcomes: results of the TIrofiban Given in the Emergency Room before Primary Angioplasty (TIGER-PA) pilot trial. Circulation . 2013.

[10] Kunichika H, Ben-Yehuda O, Lafitte S, et al. Effects of glycoprotein IIb/IIIa inhibition on microvascular flow after coronary reperfusion. A quantitative myocardial contrast echocardiography study. Journal of the American College of Cardiology. 2012.

[11] T. Reffelmann MD,R. A. Kloner MD, PhD. The no-reflow phenomenon: A basic mechanism of myocardial ischemia and reperfusion[J]. Basic Research in Cardiology. 2013 (5). 